\title{
COMPARISON OF PARTICLE SWARM OPTIMIZATION AND GENETIC ALGORITHM IN RATIONAL FUNCTION MODEL OPTIMIZATION
}

\author{
Somayeh Yavari ${ }^{\text {a }}$, Mohammad Javad Valadan Zoej ${ }^{\text {a }}$, Mehdi Mokhtarzade ${ }^{\text {a }}$, Ali Mohammadzadeh ${ }^{\text {a }}$ \\ ${ }^{a}$ K.N. Toosi University of Technology, Geodesy and Geomatics Eng. Faculty, Photogrammetry and Remote Sensing \\ Eng. Department, Tehran, Iran - yavari_se@yahoo.com; (valadanzouj,m_mokhtarzade)@kntu.ac.ir; \\ almoh2@gmail.com
}

ISPRS XXII congress, commission I/4

KEY WORDS: Rational Function Model (RFM), Particle Swarm Optimization (PSO), Genetic Algorithm (GA), Mathematical Modelling, High Resolution Satellite Images (HRSIs)

\begin{abstract}
:
Rational Function Models (RFM) are one of the most considerable approaches for spatial information extraction from satellite images especially where there is no access to the sensor parameters. As there is no physical meaning for the terms of RFM, in the conventional solution all the terms are involved in the computational process which causes over-parameterization errors. Thus in this paper, advanced optimization algorithms such as Genetic Algorithm (GA) and Particle Swarm Optimization (PSO) are investigated to determine the optimal terms of RFM. As the optimization would reduce the number of required RFM terms, the possibility of using fewer numbers of Ground Control Points (GCPs) in the solution comparing to the conventional method is inspected. The results proved that both GA and PSO are able to determine the optimal terms of RFM to achieve rather the same accuracy. However, PSO shows to be more effective from computational time part of view. The other important achievement is that the algorithms are able to solve the RFM using less GCPs with higher accuracy in comparison to conventional RFM.
\end{abstract}

\section{INTRODUCTION}

Nowadays, due to the availability of High Resolution Satellite Images (HRSIs), accurate geospatial information could be extracted from those types of images. This information can be used in different applications such as image matching, image registration, ortho-rectification, mapping and so on. For this purpose, there is a necessity of transforming satellite data from image space to ground space. The accuracy of the transformation affects the accuracy of the extracted geospatial information. The existing transformation models fall into two categories of parametric (rigorous) such as Orbital Parameter Models (Toutin, 2003; Valadan Zoej and Petrie, 1998; Valadan Zoej and Sadeghian, 2003b) and non-parametric (non-rigorous) such as Rational Function Models (Tao and Hu, 2001; Tao and $\mathrm{Hu}$, 2002; Sadeghian et al., 2001; Valadan Zoej et al., 2006; Yavari et al., 2008).

The Orbital Parameter Models reconstruct the image geometry at the time of imaging and so needs the interior orientation parameters and ephemeris data of the satellite. Hence the fundamental problem to use the HRSIs in rigorous models is disinclination of some HRSIs vendors such as GeoEye to expose the ephemeris data and interior orientation parameters of satellites. In addition, these models are sensor dependent and of course the solution of them has much complexity.

In opposite, the non-rigorous models need no interior information about the satellite, are not dependent to the sensor type, and have low computational complexity. Thus due to the mentioned advantages, non-parametric transformations are utilized for HRSIs rectification by many researchers in the field of photogrammetry and remote sensing. However it should be considered that the main deficiency of non-parametric transformations is the necessity of using significant well-defined GCPs for image rectifications in conventional terrain dependent approach. Therefore non-parametric models are very sensitive to the field topography, number and distribution of GCPs and input errors.

RFM as a non-parametric model needs a lot of accurate, welldistributed GCPs which is a time consuming and expensive process. Also the problem of over-parameterization errors exists because of using too much coefficients. In fact, coefficients in RFM do not have any physical meaning which makes it impossible to find their best combination.

In order to overcome this problem, optimization based algorithms seems to be an appropriate solution. Genetic Algorithm (Haupt and Haupt, 2004; Sastry et al., 2005) and Particle Swarm Optimization (Firsandaya Malik et al., 2007; Hu et al., 2004; Kennedy and Eberhart, 1995; Kennedy and Eberhart, 1997; Shi and Eberhart, 1998) as two important evolutionary algorithms are frequently used by the researchers to find the optimal solutions.

In this paper, the possibility of using GA and PSO are investigated to find the optimal combination of coefficients which may lead to eliminate over-parameterization errors, to reduce GCPs and to rectify HRSIs with more accuracy. Also the results are compared with conventional RFM to evaluate the algorithms. For this purpose these methods are tested on an IKONOS-Geo image with different combination of Ground Control Points (GCPs) and Independent Check Points (ICPs). Based on the results, both GA and PSO are able to determine the optimal terms of RFM to achieve rather the same accuracy. However, PSO shows to be more effective from computational time point of view. The other important achievement is that the algorithms are able to solve the RFM with higher accuracy in comparison to conventional solutions when using less GCPs. As a conclusion, both GA and PSO when using for RFM optimization, can achieve sub-pixel accuracy even with just 4 GCPs. 


\section{STUDY AREA}

This paper used an IKONOS-Geo image over Hamedan city, west of IRAN. The IKONOS-Geo image was acquired on $7 / 10 / 2000$ with an off-nadir angle of $20.4^{\circ}$ and a sun elevation of $47.4^{\circ}$. The elevation ranged between $1700 \mathrm{~m}$ and $1900 \mathrm{~m}$. In total, 58 control points are extracted from 1:1000 scale digital maps produced by the Iranian National Cartographic Center (NCC). The points are distinct features such as buildings, pool corners, walls and road junctions.

\section{RATIONAL FUNCTION MODEL}

As the Rational Function Model is the most known nonparametric model, in this section, RFM solution is described briefly. Rational Function Model (in the forward form) determines the image coordinates from the ratio of two polynomials of object coordinates as (Tao and $\mathrm{Hu}, 2001$; OGC, 1999) Eq. (1) and Eq. (2):

$r=\frac{P_{1}(X, Y, Z)}{P_{3}(X, Y, Z)}$

$c=\frac{P_{2}(X, Y, Z)}{P_{4}(X, Y, Z)}$

$P_{i}(X, Y, Z)=a_{i 0}+a_{i 1} X+a_{i 2} Y+a_{i 3} Z+a_{i 4} X Y+a_{i 5} X Z+a_{i 6} Y Z+$

$a_{i 7} X^{2}+a_{i 8} Y^{2}+a_{i 9} Z^{2}+a_{i 10} X Y Z+\ldots$

Where $(r, c)$ are normalized image coordinates and $(X, Y, Z)$ are normalized object coordinates. Most photogrammetric applications assume that $P_{3}=P_{4}$.

The process of normalization in both image and object coordinates is very important in RFM. This causes less computational input error and numeric stability, leading to better accuracy (Tao and $\mathrm{Hu}, 2001$ ). RFM can be solved on two methods: Terrain-Independent (use the satellite parameters and sensor model with no GCPs) and Terrain-Dependent (that use only GCPs to compute the unknown parameters). Since for Geo-rectified images (such as IKONOS-Geo) precise ancillary data is not available, the present paper only investigates the Terrain-Dependent direct forward RFM. Because all coefficients are used in solving the conventional approach process, it has its' own disadvantages like: necessity of using too many GCPs, increasing the probability of over parameterization errors, tendency to inaccurate results (Tao and $\mathrm{Hu}, 2001)$. So to overcome these problems, we assess the potential of two evolutionary algorithms (GA and PSO) in finding the effective parameters of RFM. Hence a brief description about these two methods is mentioned bellow.

\subsection{Optimization of the RFM by GA}

GA is a meta-heuristic approach that attempt to simulate the natural evolution process and optimize the desired function using some natural evolutional operators such as mutation, crossover and selection (Haupt and Haupt, 2004; Sastry et al., 2005). In GA, a population of candidate solutions that called chromosomes is initialized with random values. In order to find the optimal terms, presence or absence of a specific term in an RFM solution can be represented by 1 or 0 in binary GA respectively. A sample of chromosomes used in GA for RFM solution (Valadan Zoej et al., 2007) is shown in Figure 1.

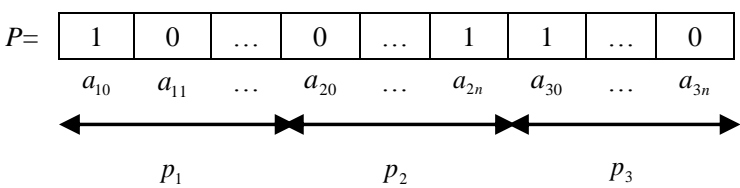

Figure 1. A chromosome scheme used in binary GA

Based on this chromosome, the first coefficient is used to solve the RFM but the second not and so on.

Then the fitness values are calculated using the cost function (1/RMSE), and afterwards the evolutional operators of selection, crossover and mutation are applied on initial chromosomes (based on fitness value of each chromosome) to produce offspring chromosomes. The GA parameters are set as Table 1 .

Table 1

Parameters of the GA used in RFM optimization

\begin{tabular}{|c|c|}
\hline Population size & 50 \\
\hline Chromosomes length & 32 bit \\
\hline Selection method & Tournament selection \\
\hline Crossover method & uniform crossover \\
\hline mutation probability & $25-35 \%$ of all chromosomes \\
\hline Elitism Number & 2 \\
\hline
\end{tabular}

This process iterates until reaching to a stopping criteria. A gene is said to have converged when $95 \%$ of the population share the same value. The population is said to have converged when all of the genes have converged. Finally the chromosome that has highest fitness is chosen as the optimized solution. In RFM optimization using GA, the proposed algorithm in (Valadan Zoej et al., 2007) is used.

\subsection{Optimization of the RFM by PSO}

PSO was originally designed and introduced by Eberhart and Kennedy in 1995 based on social intelligence of a group of birds or fishes (Kennedy and Eberhart, 1995; Kennedy and Eberhart, 1997; Shi and Eberhart, 1998). In binary PSO, a population (swarm) of birds (possible solutions or individuals or particles) is initialized randomly with values of $\{0,1\}$. It means each particle is a combination of one and zero which indicate the presence or absence of corresponding coefficient in the cost function respectively (similar to the chromosome scheme shown in Figure 1). These particles are represented as the current positions $(p)$. Then the fitness values of these particles are calculated using the cost function of direct forward RFM. Based on these fitness scores, the best positions of each particle (Pbest) and the global best position of all particles (Gbest) are determined.

The 1/RMSE of ICPs for each particle is considered to be its fitness value. In an iterative process, the velocity of each particle $(v)$ is updated as below (3).

$$
\begin{aligned}
& v_{i j}(t+1)=w(t) \cdot v_{i j}(t)+c_{1} \cdot r_{1} \cdot\left[\operatorname{GBest}_{i}(t)-p_{i j}(t)\right]+ \\
& c_{2} \cdot r_{2} \cdot\left[\operatorname{PBest}_{i}(t)-p_{i j}(t)\right]
\end{aligned}
$$

where $\quad i$ is the index of particle in the population; $j$ is the index of bits in the binary string of each particle; $t$ is the iteration number; 
$r_{1}$ and $r_{2}$ are two uniform random values in $[0,1]$;

$c_{1}$ and $c_{2}$ are two constant acceleration coefficients and $w(t)$ is time varying inertia weight.

A nonlinear inertia weight (w) (Umapathy et al., 2010) is used to adjust the effect of the current velocities in computation of the new velocity values (4).

$w(t)=w_{\min }+\left(w_{\max }-w_{\min }\right) \frac{t_{\max }-t}{t}$

In the above equation, $w_{\max }$ and $w_{\min }$ are two constant experimental parameters, and $t_{\max }$ is the maximum number of iterations.

In binary PSO, the velocities of the particles are rather defined in terms of probabilities that a bit will change to one. Using this definition a velocity must be restricted within the range $[0,1]$. So a map is introduced to map all real valued numbers of velocity to the range $[0,1]$. Usually sigmoid function is chosen as the normalization function (Kennedy and Eberhart, 1997; Yang et al., 2004):

$p_{i j}(t+1)=\left\{\begin{array}{cc}1, \text { if } & r_{i j}<\operatorname{sig}\left(v_{i j}(t)\right) \\ 0, & \text { otherwise }\end{array}\right.$

$r_{i j}$ is a uniform random number in $[0,1]$.

As can be seen from above equations, it's obvious that in spite of other evolutionary methods like GA, this method doesn't employ any complex evolutionary operators such as selection; crossover and mutation hence the computational time should be lower than GA in theory.

In this paper, the maximum number of terms in $P_{1}, P_{2}$ and $P_{3}$ is set to 11,11 , and 10 , respectively, which means that a total of 32 terms are used in the optimization process $\left(a_{30}=1\right)$.

The PSO parameters used in this research are shown in Table 2. A population size of 30 is chosen as the minimum number of particles where required accuracies are obtained in a reasonable computational time.

Table 2

Parameters of the PSO used in RFM optimization

\begin{tabular}{|c|c|c|}
\hline \multicolumn{2}{|c|}{ Population size } & 30 \\
\hline \multirow{2}{*}{$\mathrm{v}$} & $v_{\max }$ & 3 \\
\cline { 2 - 3 } & $v_{\min }$ & -3 \\
\hline \multirow{3}{*}{$\mathrm{W}$} & $w_{\max }$ & 1 \\
\cline { 2 - 3 } & $w_{\min }$ & 0.02 \\
\cline { 2 - 3 } & $t_{\max }$ & 200 \\
\hline \multicolumn{2}{|c|}{$c_{1}$} & 1.5 \\
\hline \multicolumn{2}{|c|}{$c_{2}$} & 1.5 \\
\hline
\end{tabular}

The parameters $v_{\max }, v_{\min }, w_{\max }, w_{\min }, t_{\max }, c_{1}$ and $c_{2}$ are selected based on (Firsandaya Malik et al., 2007; Kennedy and Eberhart, 1997; Shi and Eberhart, 1998) and also experimentally to balance the global and local search (randomness and intelligence) of the PSO. However it should be noticed that based on our experimental results, PSO is rather stable to the mild changes of these parameters.

The termination of the PSO is controlled by both the maximum number of generations (i.e., ${ }^{t_{\max }}$ ) and by a convergence criterion. This criterion is satisfied when the maximum difference between the fitness of GBest, the mean of PBest and the mean of the particle fitness are less than a threshold. The appropriate threshold value is determined empirically and depends on the accuracy of GCPs and also the image spatial resolution. As a result, this threshold is set to 10 for IKONOS image. However, different values in the range of 10 to 100 are tested and the results show that this value does not have a significant effect on the final results. The mean of PBest and the particle fitness are computed over the best half of the population to prevent the undesired effects of blunder individuals.

\section{EXPERIMENTAL RESULTS AND DISCUSSIONS}

To evaluate these methods, different combinations of welldistributed GCPs and ICPs are used.

All of these optimization algorithms are performed ten times to assess the stability of the results. It should be mentioned that not only the arrangement of selected terms but also the number of these terms are different in different runs. However their accuracies are rather comparable (see 3rd and 4th columns of Table 3). Among them, the best one is selected which is presented in Table 3.

As shown in Table 3, PSO and GA-optimized RFM models show almost similar accuracy values, while PSO models are faster. The longer computational time of GA model can be due to the larger population size required in order to achieve the same accuracy as PSO model.

Also both methods can optimize the RFM to obtain sub-pixel accuracy for IKONOS image when as few as four GCPs are used. Residual vector plot diagrams show that no systematic errors are occurred.

Furthermore a complete series of polynomials in conventional RFM based on (Tao and Hu, 2001; Yavari et al., 2008) with different combinations of GCPs and ICPs is used and showed in Table 4. According to this table, using complete series of polynomials like (Tao and $\mathrm{Hu}, 2001$ ) requires a large number of GCPs while in the proposed methods, better results are obtainable using much less GCPs.

Due to a lack of degrees of freedom, conventional RFMs cannot be solved when only 4 or 5 GCPs are available. The comparison between the results obtained from both conventional RFMs and RFMs optimized with PSO and GA demonstrate the efficiency of these methods proposed in this paper. 
International Archives of the Photogrammetry, Remote Sensing and Spatial Information Sciences, Volume XXXIX-B1, 2012 XXII ISPRS Congress, 25 August - 01 September 2012, Melbourne, Australia

TABLE 3

RFM results obtained by Genetic Algorithm and conventional PSO optimization over an IKONOS-Geo image

\begin{tabular}{|c|c|c|c|c|c|c|c|c|}
\hline \multirow[b]{2}{*}{$\begin{array}{l}\text { Optimization } \\
\text { Algorithm }\end{array}$} & \multirow[b]{2}{*}{ GCPs \& ICPs } & \multirow[b]{2}{*}{$\begin{array}{c}\text { Mean RMSE } \\
\text { of ICPs in } 10 \\
\text { runs } \\
\text { (in pixel) }\end{array}$} & \multirow{2}{*}{$\begin{array}{l}\text { Standard } \\
\text { Deviation of } \\
\text { RMSE of } \\
\text { ICPs }\end{array}$} & \multicolumn{5}{|c|}{ Optimum Coefficients obtained by optimization methods } \\
\hline & & & & $\begin{array}{l}\text { Number of } \\
\text { coefficients } \\
P_{1}, P_{2}, P_{3 *}\end{array}$ & $\begin{array}{l}\text { RMSE of GCPs } \\
\text { (in pixel) }\end{array}$ & $\begin{array}{l}\text { RMSE of ICPs } \\
\quad \text { (in pixel) }\end{array}$ & $\begin{array}{l}\text { Performance } \\
\text { time }(\mathrm{s}) * *\end{array}$ & iterations \\
\hline \multirow{4}{*}{$\begin{array}{l}\text { Conventional } \\
\text { PSO }\end{array}$} & $30 \& 28$ & 0.62 & 0.018 & $6,10,4$ & 0.55 & 0.60 & 1876 & 109 \\
\hline & $10 \& 48$ & 0.72 & 0.023 & $3,7,3$ & 0.66 & 0.68 & 1781 & 200 \\
\hline & $5 \& 53$ & 1.06 & 0.116 & $4,3,3$ & 0 & 0.92 & 709 & 200 \\
\hline & $4 \& 54$ & 1.14 & 0.109 & $4,4,0$ & 0 & 1.02 & 489 & 200 \\
\hline \multirow{4}{*}{ GA } & $30 \& 28$ & 0.60 & 0.011 & $7,7,4$ & 0.53 & 0.59 & 4240 & 137 \\
\hline & $10 \& 48$ & 0.68 & 0.031 & $4,8,2$ & 0.63 & 0.64 & 1483 & 121 \\
\hline & $5 \& 53$ & 0.84 & 0.068 & $2,4,2$ & 0.37 & 0.75 & 764 & 152 \\
\hline & $4 \& 54$ & 1.08 & 0.241 & $3,3,1$ & 0.36 & 0.89 & 795 & 211 \\
\hline
\end{tabular}

$* a_{30}=1$ and it is excluded from the number of terms in $p_{3}$.

TABLE 4

Complete series of polynomials in conventional RFM over an IKONOS-Geo image using different combinations of GCPs and ICPs

\begin{tabular}{|c|c|c|c|c|c|c|}
\hline \multirow{2}{*}{ GCPs \& ICPs } & \multicolumn{3}{|c|}{ RMSE of GCPs (in pixel) } & \multicolumn{3}{|c|}{ RMSE of ICPs (in pixel) } \\
\hline & $\Delta \mathrm{x}$ & $\Delta \mathrm{y}$ & $\Delta x y$ & $\Delta \mathrm{x}$ & $\Delta \mathrm{y}$ & $\Delta x y$ \\
\hline $30 \& 28$ & 0 & 0 & 0 & 5.21 & 10.95 & 12.13 \\
\hline $35 \& 23$ & 0.02 & 0.01 & 0.03 & 3.97 & 0.97 & 4.08 \\
\hline $40 \& 18$ & 0.02 & 0.02 & 0.03 & 1.17 & 0.79 & 1.41 \\
\hline
\end{tabular}

\section{CONCLUSION}

In this paper, a comparison of the results obtained using the optimization methods (GA and PSO) with those obtained using traditional RFMs demonstrate the efficiency of both algorithms in terms of both the accuracy and the number of required GCPs. As a conclusion, both GA and PSO when using for RFM optimization, can achieve sub-pixel accuracy even with just 4 GCPs.

\section{References}

[1] R. Firsandaya Malik, Th. Abdul Rahman, S. Hashim, and R. Ngah, "New particle swarm optimizer with sigmoid increasing inertia weight," Int. J. Comput. Sci. and Security, vol. 1, no. 2, pp. 35-44, 2007.

[2] R. L. Haupt and S. E. Haupt, Practical Genetic Algorithm, $2^{\text {nd }}$ Ed. New Jersey: Wiley, 2004.

[3] X. Hu, Y. Shi and R. C. Eberhart, "Recent advances in particle swarm," in Proc. IEEE Congr. Evol. Computation, vol. 1, Portland, USA, 2004, pp. 90- 97.

[4] J. Kennedy and R. C. Eberhart, "Particle swarm optimization," in Proc. IEEE Int. Conf. Neural Networks, vol.4, Perth, WA, 1995, pp. 1942-1948.

[5] J. Kennedy and R. C. Eberhart, "A discrete binary version of the particle swarm algorithm," in IEEE Int. Conf. Syst., Man and Cybern., Computational Cybern. Simulations, vol. 5, Orlando, 1997, pp. 4104-4108.

[6] C. V. Tao and Y. Hu, "A comprehensive study of the rational function model for photogrammetric processing," Photogrammetric Eng. \& Remote Sensing, vol. 67, no. 12, pp. 1347-1357, Dec. 2001.

[7] C. V. Tao and Y. Hu, "3D construction methods based on the Rational Function Model photogrammetric processing," Photogrammetric Eng. \& Remote Sensing, vol. 68, no. 7, pp. 705-714, Jul. 2002.

[8] S. Sadeghian, M. J. Valadan Zoej, M. R. Delavar, and A. Abootalebi, "Precision rectification of high resolution satellite imagery without ephemeris data," Int. J. Appl. Earth Observation and Geoinformation (JAG), vol. 3, no. 4, pp. 366-371, 2001.
[9] K. Sastry, D. Goldberg, and G. Kendall, "Genetic Algorithm," in Search Methodologies, Springer, 2005, Ch. 4, pp. 97-105.

[10] Y. Shi and R. Eberhart, "A modified particle swarm optimizer," in 1998 IEEE Int. Conf. Evol. Computation Proc., Anchorage, AK, pp. 69-73.

[11] Toutin, Th., spring 2003, Review Paper: Geometric Processing of Remote Sensing Images: Models, Algorithms, and Methods. International Journal of Remote Sensing, 24.

[12] P. Umapathy, C. Venkataseshaiah, and M. Senthil Arumugam, "Particle swarm optimization with various inertia weight variants for optimal power flow solution," Discrete Dynamics in Nature and Soc., vol. 2010, Article ID 462145, 15 pages, 2010. doi:10.1155/2010/462145.

[13] M. J. Valadan Zoej, M. Mokhtarzadeh, A. Mansourian, H. Ebadi, and S. Sadeghian, "Rational function optimization using genetic algorithms," Int. J. Appl. Earth Observation and Geoinformation (JAG), vol. 9, no. 4, pp. 403-413, 2007.

[14] M. J. Valadan Zoej and G. Petrie, "Mathematical modeling and accuracy testing of SPOT level 1B stereo pairs," Photogrammetric Rec., vol. 16, no. 91, pp. 67-82, Apr. 1998.

[15] M. J. Valadan Zoej and S. Sadeghian, "Orbital parameter modeling accuracy testing of Ikonos Geo image," Photogrammetric J. Finland, vol. 18, no. 2, pp. 70-80, 2003b.

[16] M. J. Valadan Zoej, S. Yavari, and S. Sadeghian, "Mathematical modeling of Ikonos-Geo image," presented at the ISPRS Workshop: Topographic mapping from space, vol. XXXVI-1/W41, Ankara, 2006.

[17] S. Yang, M. Wang and L. Jiao, "A quantum particle swarm optimization," in Proc. IEEE Congr. Evol. Computation, New Jersey, 2004, pp. 320-324.

[18] S. Yavari, M. J. Valadan Zoej and S. Sadeghian, "Mathematical modeling of Geo-rectified dynamic space images," Int. J. Geoinformatics, vol. 4, no. 4, pp. 47-55, Dec. 2008. 